\title{
Development of 3-Dimensional Model of Femur Bone Considering Cortical and Cancellous Structures
}

\author{
${ }^{1}$ Mohd Amir Shahlan Mohd Aspar, ${ }^{2}$ Nasrul Anuar Abd Razak, ${ }^{3}$ Mohammed Rafiq \\ Abdul Kadir and ${ }^{1}$ Hadi Manap \\ ${ }^{1}$ Faculty of Engineering Technology, University Malaysia Pahang, 26300 Kuantan, \\ Pahang, Malaysia \\ ${ }^{2}$ Department of Biomedical Engineering, Faculty of Engineering, University of Malaya, \\ 50603 Kuala Lumpur, Malaysia \\ ${ }^{3}$ Faculty of Biosciences and Medical Engineering, Universiti Teknologi Malaysia, 81310 \\ Johor Bahru, Malaysia \\ amirshahlanaspar@gmail.com,nasrul.anuar@um.edu.my,rafiq@biomedical.utm.myand \\ hadi@ump.edu.my,
}

Abstract- Femur bone is commonly used for various experimental and computer simulation works in multidiscipline research. Various techniques are applied prior to mimic the actual bone properties. In order to perform any research related to human bone, some issues need to take into account such as cost, ethical concern and limited bone sample availability. Experimental test and computer simulation related to femur bone model commonly executed using hollow cylinder, solid rod or beam elements instead of real anatomy. The aims of present study is to provide $3 D$ femur bone model construction considering both cortical and cancellous structures utilising only one software approach. The constructed model could be utilised for various research purposes such as computer simulation, 3D print of bone model and experimental test. Complete femur bone model which include proximal, shaft and distal condyles is successfully constructed and ready to be used for further investigation. Mimics software was the only software used in present study to performed overall task.

Keywords- Femur Cortical, femur cancellous, bone model construction

\section{Introduction}

The femur is a long bone located in between hip and knee joints. Femur is the only bone in the thigh that act upon extreme forces during many activities, such as running, jumping, walking, and standing $[1,2]$. Femur measured as the longest, heaviest and strong bone in the body [3], supported with the muscles of hip and thigh.

Femur bone is commonly used for various experimental and computer simulation works in multidiscipline research including orthopaedic surgery, biomechanics engineering, biomedical engineering, rehabilitation and medical implant technologies. Various techniques 
are applied prior to mimic the actual bone properties. In multiple software application [4], 3-dimensional (3D) femur bone model was constructed through the creation of polygonal model from computed tomography (CT) data and exported to CAD software for bone surfaces creation. However, the limitation to develop real skeletal structure which includes hard and soft tissues could not be implemented. In laser scan approach [5], CAD model was obtained based on external geometric features of femur bone. The dimension of marrow cavity was created through approximation of 1.6 $\mathrm{cm}$ hollow cylinder using design software (solidworks, Dassault Systems Solidworks Corp., USA). The simple cylindrical rod representing long bone fracture for computer simulation was reported as well $[6,7]$. In experimental works, utilisation of cylindrical rod [610] and synthetic bone [11] to mimic long bone structures were implemented. 3D beam elements were used to model the proximal and distal of long bone to analyse the fracture fixation of external fixator system [12]. In another experiment, ultra high molecular weight polyethylene (UHMWPE) hollow cylinder with an outer diameter of 5.08 $\mathrm{cm}$ and inner of $2.54 \mathrm{~cm}$ was used to simulate knee-spanning external fixation [8].

In order to perform analysis related to human bone, some issues need to take into account such as cost, ethical concern and limited bone sample availability. As a result, prolonged experimental waiting period and unconformity research outcome are experienced. Experimental test and computer simulation related to femur bone model commonly executed using hollow cylinder, solid rod or beam elements instead of real anatomy. The actual bone anatomy and mechanical properties almost totally neglected which effect on reliability and accuracy of the outcome.

The aims of present study is to provide 3D femur bone model construction considering both cortical and cancellous structures utilising only one software (Mimics, Materialise, Leuven, Belgium) approach. The constructed bone model could be utilised for various research purposes such as computer simulation, 3D print of bone model and experimental test.

\section{Material and Methods}

In this project, a healthy female subject was scanned to generate CT images of the lower limb. Then, the femoral bone was constructed from the CT images datasheet using Mimics (Materialise, Leuven, Belgium) software. To begin, the DICOM CT images were imported into Mimics software. The software capable to visualise the CT images in three different views include axial, sagittal and coronal planes. The cortical and cancellous bones were segmented to construct the 3D model of femur. In clinical practice, CT image of hard tissues (bone) are visualised in bright color while soft tissue (skin, ligaments, muscles and tendons) could not be visualised which represent in dark color. The darker color of component visualised, the lesser the density.

In order to separate the construction of cortical and cancellous bones, different range of Hounsfield value are applied. The cortical bone is extracted by means of thresholding using value range from 750 Hounsfield units 
(HU) to $3071 \mathrm{HU}$. For cancellous bone, extraction by means of thresholding using value range from $200 \mathrm{HU}$ to $750 \mathrm{HU}$ was performed. Separate masks on cortical and cancellous bones are then obtained. The correction of mask is required prior to obtain solid 3D model. This could be
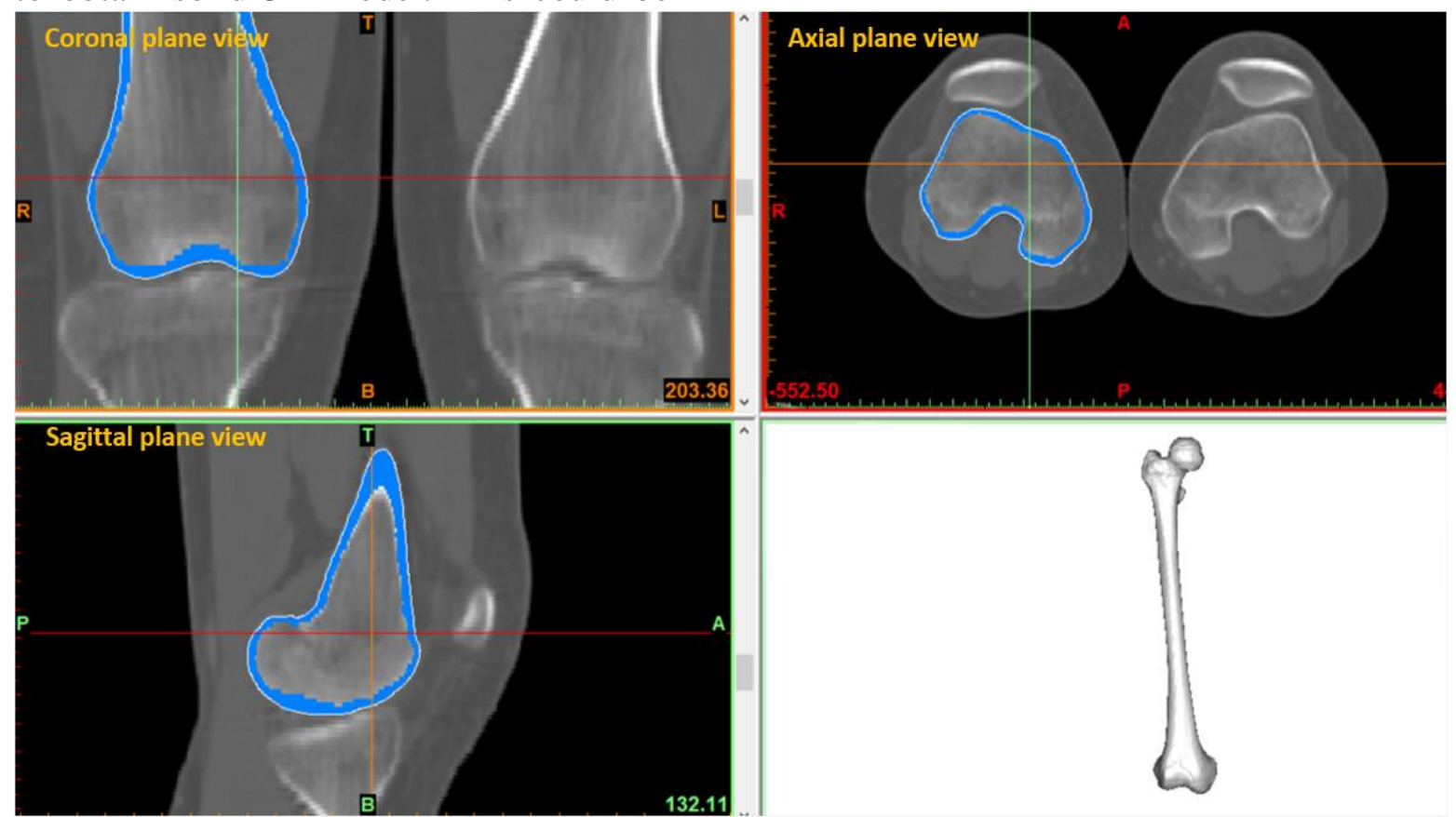

Figure 1: Construction of femur cortical bone done by addition of mask and subtraction of unnecessary mask generated during threshold value application. Figure 1 and Figure 2 illustrate the completed of mask generation for both cortical and cancellous bones. 

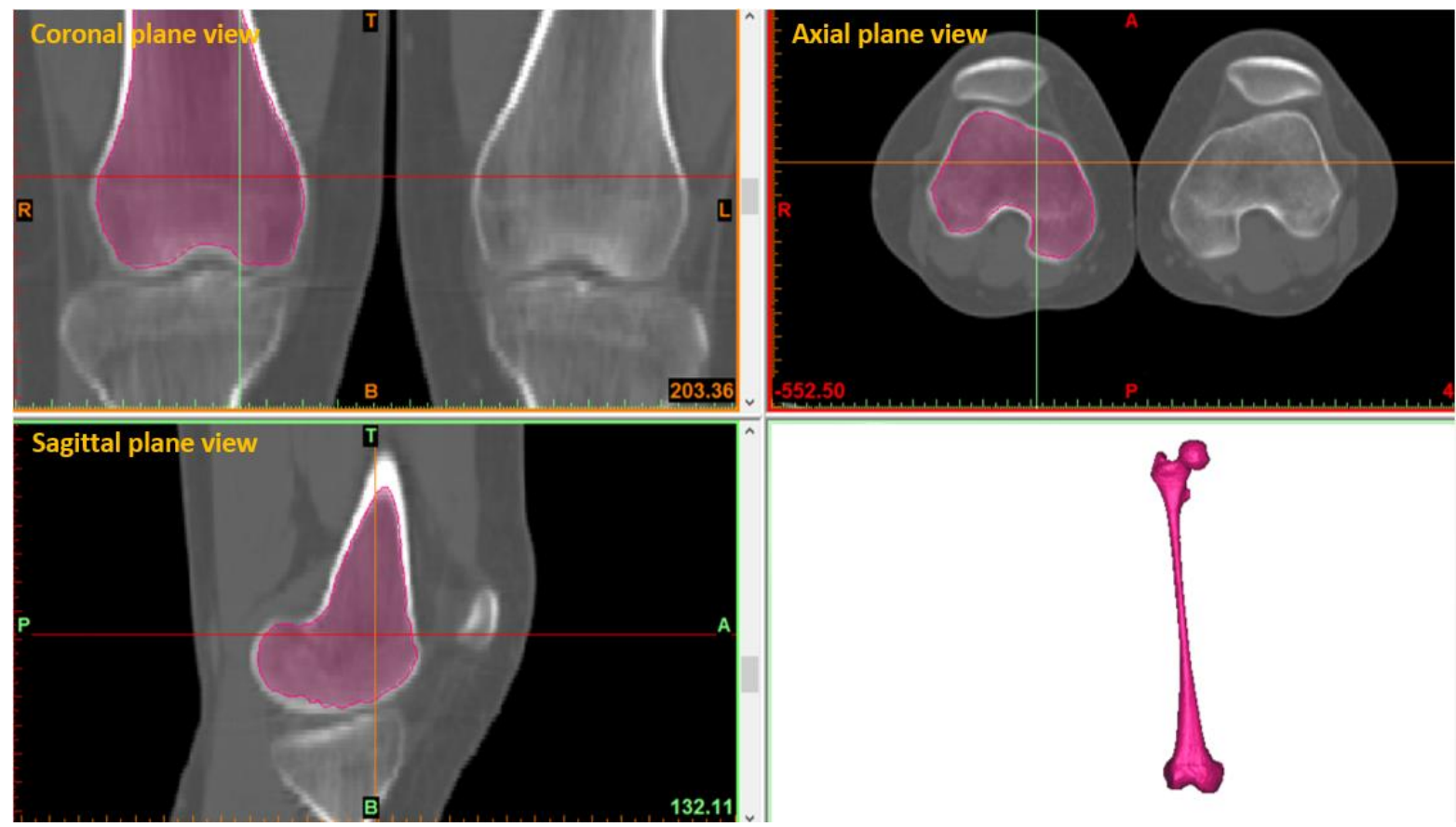

Figure 2: Construction of femur cancellous bone

Then, by using calculate tools provided in the software, cortical and cancellous bones were successfully

\section{Step 1: CT scan}
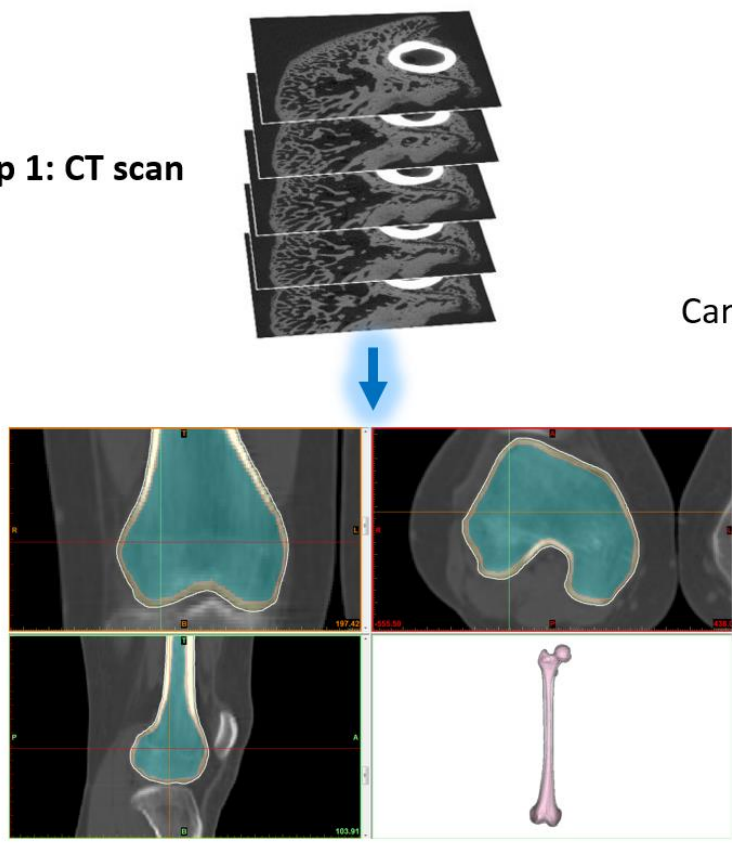

Step 2: Femur bone construction constructed. The overall steps performed to construct 3D model of femur bone are illustrated in figure 3 .
Step 3:

3D Femur bone model

Figure 3: Complete 3D femur bone construction 


\section{Result}

Mimics software was fully utilised in the present study to construct 3D femur bone. Both cortical and cancellous bones were considered in the construction using CT images (Figure 4). Complete femur bone model which include proximal, shaft and distal condyles is successfully constructed and ready to be used for further investigation. Mimics software was the only software used in present study to performed overall task.
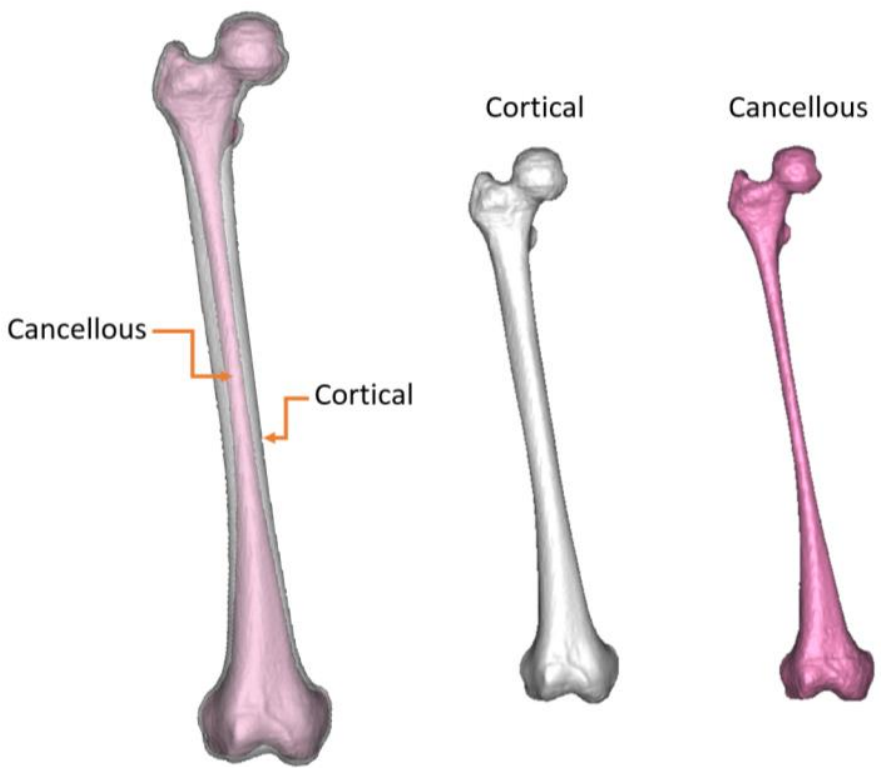

Figure 4: Complete model construction of femur considering cortical and cancellous bones based on real anatomy

\section{Discussion}

Used real femur bone anatomy instead of approximation model

Previous studies demonstrate various methods to develop bone structure for analyses. Hollow cylinder, solid rod and beam element are some of the approaches [5-7, 9]. In present study, instead of using structural approximation, we revealed simple technique on developing bone structure based on real anatomy. CT images in DICOM format has been successfully utilised on bone construction which considering both cortical and cancellous structures. The complete bone construction are capable to be exported into various file formats such as stl, igs, iges and dcb. By applying the method proposed in present study, it helps researchers to construct bone component faster, easier and simpler. In addition, the better reliability and accuracy of outcome is expected when using actual bone anatomy rather than approximation structures. 


\section{Simpler step in model construction}

In present study, we have successfully develop 3D model of femur bone using only one software approach. Any imaging modalities such as CT, MRI and $\mathrm{x}$-ray data is required as a basis for model construction. Previous studies reported of bone construction using more than one software or methods approach $[1,10]$. Some required additional steps such as laser scan and creation of additional structure to represent the cancellous bone [5]. Others used only cortical structure to represent bone in analyses [11-14]. Present study provide simpler step on 3D bone model construction which could be utilised for further investigation related to human bone. 3D printing technology and computer simulation software can simply use the model produced as it can be converted into various file format.

Ethical concern, cost, prolonged experimental waiting period and outcome unconformity

Ethical concern and cost are the most common issues while considering human or animal as a sample in experimental work. There is no other way to avoid the ethical concern instead of dealing with it. For cost, it can be reduced if the sample is already available to be use or easily accessible in lab. But the majority will experience difficulty in term of cost and effort on getting the bone sample for analysis purpose. In present study, we have revealed simple methods on femur bone construction using CT datasheets. The methods for bone construction is easier. Only medical imaging data and single software utilisation are required tools. In addition, in case of mechanical structure test, this method offers repetition of bone model production through the use of 3D printer. Researchers are able to perform experiment test up to hundred times of repetition until optimum result is obtain. The issue of prolonged experimental waiting period and unconformity research outcome could also be avoided.

\section{Conclusion}

The model of 3D femur bone with cortical and cancellous structures was successfully constructed. Mimics software was the only software required to perform the overall task. The 3D bone model produced is able to be utilised for various purpose of research investigation such as computer simulation and experiment test.

\section{References}

[1] S. Das and S. K. Sarangi, "Finite Element Analysis of Femur Fracture Fixation Plates," Int. J. Basic Appl. Biol., vol. 1, no. 1, pp. 1-5, 2014.

[2] D. G. Ju, P. J. Mogayzel, P. D. Sponseller, F. Familiari, and E. G. McFarland, "Bilateral midshaft femoral fractures in an adolescent baseball player," J. Cyst. Fibros., vol. 15, no. 4, pp. 41-43, 2016.

[3] A. Dhanopia and M. Bhargava, "Finite Element Analysis of Human Fractured Femur Bone Implantation with PMMA Thermoplastic Prosthetic Plate," Procedia Eng., vol. 173, pp. 16581665, 2017.

[4] S. Vulovic, N. Korunovic, M. 
Trajanovic, N. Grujovic, and N. Vitkovic, "Finite element analysis of CT based femur model using finite element program PAK," J. Serbian Soc. Comput. Mech., vol. 6, no. 2, pp. 160-166, 2012.

[5] U. N. Mughal, H. A. Khawaja, and M. Moatamedi, "Finite element analysis of human femur bone," Int. J. Multiphys., vol. 9, no. 2, pp. 1-10, 2015.

[6] M. A. Watson, K. J. Mathias, N. Maffulli, D. W. L. Hukins, and D. E. T. Shepherd, "Finite element modelling of the Ilizarov external fixation system," Proc. Inst. Mech. Eng. H., vol. 221, no. 8, pp. 863 71, 2007.

[7] M. B. Sternick, D. Dallacosta, D. Á. Bento, and M. L. do Reis, "Relationship Between Rigidity of External Fixator and Number of Pins: Computer Analysis Using Finite Elements," Rev. Bras. Ortop. (English Ed., vol. 47, no. 5, pp. 646-650, 2012.

[8] S. Strebe, H. Kim, J. P. Russell, A. H. Hsieh, J. Nascone, and R. V. O'Toole, "Analysis of strategies to increase external fixator stiffness: Is double stacking worth the cost?," Injury, vol. 45, no. 7, pp. 1049-1053, 2014.

[9] K. Stoffel, U. Dieter, G. Stachowiak, A. Gächter, and M. S. Kuster, "Biomechanical testing of the LCP - How can stability in locked internal fixators be controlled?," Injury, vol. 34, no. SUPPL. 2, 2003.

[10] K. Karunratanakul, J. Schrooten, and H. Van Oosterwyck, "Finite element modelling of a unilateral fixator for bone reconstruction: Importance of contact settings," Med. Eng. Phys., vol. 32, no. 5, pp. 461-467, 2010.

[11] U. S. Mani, C. T. Sabatino, S. Sabharwal, D. J. Svach, A. Suslak, and F. F. Behrens, "Biomechanical comparison of flexible stainless steel and titanium nails with external fixation using a femur fracture model.," J. Pediatr. Orthop., vol. 26, no. 2, pp. 182-7, 2006.

[12] T. K. K. Koo et al., "Stiffness Analysis of Dynafix External Fixator System," in Summer Bioengineering Conference, 2003, pp. 1227-1228.

[13] A. E. Yousif and M. Y. Aziz, "Biomechanical Analysis of the human femur bone during normal walking and standing up," IOSR $J$. Eng., vol. 2, no. 8, pp. 2250-3021, 2012.

[14] V. Sivakumar and A. R. U. N, "Non-Linear 3D Finite Element Analysis of the Femur Bone," Int. J. Res. Eng. Technol., vol. 2, no. 3, pp. 266-275, 2013.

[15] A. Gupta and K. M. Tse, "Finite Element Analysis on Vibration Modes of Femur Bone," in International Conference on Advances in Mechanical Engineering AETAME, 2013, pp. 827-831.

[16] D. B. Kumar and K. G. Muthurajan, "Finite Element Analysis of External Ilizarov Ring and Hybrid Fixators," Int. J. Sci. Res., vol. 3, no. 2, pp. 76-78, 2014. 
INTERNATIONAL JOURNAL OF ENGINEERING TECHNOLOGY AND SCIENCES (IJETS) Vol.7 (1) June 2017 DOI: http://dx.doi.org/10.15282/ijets.7.2017.1.12.1074 